Sharif University of Technology
Scientia Iranica
SCIENTIA
I RAN I CA

\title{
Dispersion of surface waves in a transversely isotropic half-space underlying a liquid layer
}

\author{
A. Bagheri ${ }^{\mathrm{a}}$, A. Khojasteh ${ }^{\mathrm{b}}$, M. Rahimian ${ }^{\mathrm{a}, *}$ and R. Attarnejad ${ }^{\mathrm{a}}$ \\ a. School of Civil Engineering, College of Engineering, University of Tehran, P.O. Box 11155-4563, Tehran, Iran. \\ b. School of Engineering Science, College of Engineering, University of Tehran, P.O. Box 11155-4563, Tehran, Iran. \\ Received 23 November 2014; received in revised form 23 August 2015; accepted 28 November 2015
}

\author{
KEYWORDS \\ Surface wave; \\ Dispersion; \\ Liquid; \\ Transversely isotropic; \\ Rayleigh wave; \\ Stoneley wave; \\ Scholte wave; \\ Frequency equation; \\ Anisotropy.
}

\begin{abstract}
Surface waves dispersion is studied in a two-layer half-space consisting of a finite liquid layer overlying a transversely isotropic solid half-space. A couple of complete potential functions are utilized to uncouple the equation of motion of the transversely isotropic solid along with a displacement potential for the liquid. The frequency equation and velocity dispersion curves are developed. Several solid materials are considered for the bed, and both phase and group velocity curves are calculated. Higher modes are also discussed and the respective curves are plotted. Various special cases are considered by letting the liquid layer depth take zero value, to form a solid half-space, or very large values to form a full-space bi-material. Moreover, an isotropic bed material can be obtained as a special case by appropriately setting the respective elastic constants. Reduced frequency equations and numerical results are derived for each case to confirm the results with existing ones.

(C) 2016 Sharif University of Technology. All rights reserved.
\end{abstract}

\section{Introduction}

Propagation of elastic surface waves in various media has been of importance and interest in different fields of science such as seismology, earthquake engineering, material engineering, geophysical studies, etc. The media consisting of a liquid layer lying over an isotropic solid half-space has been studied by scientists interested in fields such as ocean engineering, etc. [1-3].

However, in a wide range of cases, there are observations revealing the existence of anisotropy in Earth's crust. It is believed that the fact of following a preferred pattern instead of random orientations by materials depositing in the water and also existence of vertically aligned microcracks result in the transverse

\footnotetext{
*. Corresponding author. Tel.: +982161112256; Fax: +982188078263

E-mail addresses: a.h.bagheri@ut.ac.ir (A. Bagheri); akhojasteh@ut.ac.ir (A.Khojasteh); rahimian@ut.ac.ir (M. Rahimian); attarnjd@ut.ac.ir (R. Attarnejad)
}

isotropy of the geologic materials [4]. This anisotropy can considerably affect the dispersive properties of surface waves. Studying wave propagation in transversely isotropic solids is the research subject of many investigators due to its importance in different fields of science and technology. Stoneley [5] was the first to point that transverse isotropy significantly influences the wave propagation in a medium in comparison with isotropic one. Later, elastodynamics of transversely isotropic media was subsequently studied by Synge [6], Buchwald [7], and Payton [8].

Rahimian et al. [9] presented an efficient analytical formulation to obtain the response of a threedimensional transversely isotropic half-space to a timeharmonic surface loading. Khojasteh et al. [10] extended the previous work to present explicit expressions for the three-dimensional dynamic Green's functions in a transversely isotropic half-space and investigated the wave propagation. They continued the work for a two-layer half-space [11] as well. Also, Khojasteh et al. subsequently studied wave propagation in 
transversely isotropic bi-materials, tri-materials, and a multilayered half-space [12-14].

Surface waves dispersion in a model of a liquid layer overlying a transversely isotropic half-space has been investigated in some papers. Abubakar and Hudson [15] studied the dispersive properties of surface waves in such a system in the two-dimensional case. Sharma et al. [16,17] obtained the frequency equation of a three-layer model consisting of a liquid layer lying over poroelastic and anisotropic layers. Sharma [18] also tackled the dispersion of Stoneley waves in an oceanic crust model. In addition to Kumar and Miglani [19], Kumar and Kumar [20] solved the problem for the proposed oceanic crust models. In this paper, the dispersive characteristics of a three-dimensional earth crust model, consisting of a homogeneous transversely isotropic elastic media overlaid by a homogeneous compressible liquid layer, are investigated utilizing the potential method presented by Rahimian et al. [9] and extended by Khojasteh et al. [10]. The velocity dispersion curves of surface waves are plotted. Arrival times of the waves are distinctly dependent on the elastic properties of the bed. Analyzing this phenomenon to realize the earth crust's characteristics by reverse calculation is of significant importance in marine seismology and underground geophysical explorations. The formulation can also be utilized to derive Green's functions of stress and displacement fields for various load types in such a system which is applicable to earthquake engineering.

\section{Statement of the problem and governing equations}

The geometry of the system and the coordinate axes selected for the problem are shown in Figure 1. For the homogeneous transversely isotropic half-space, the time-harmonic equations of motion are expressed as [9]:

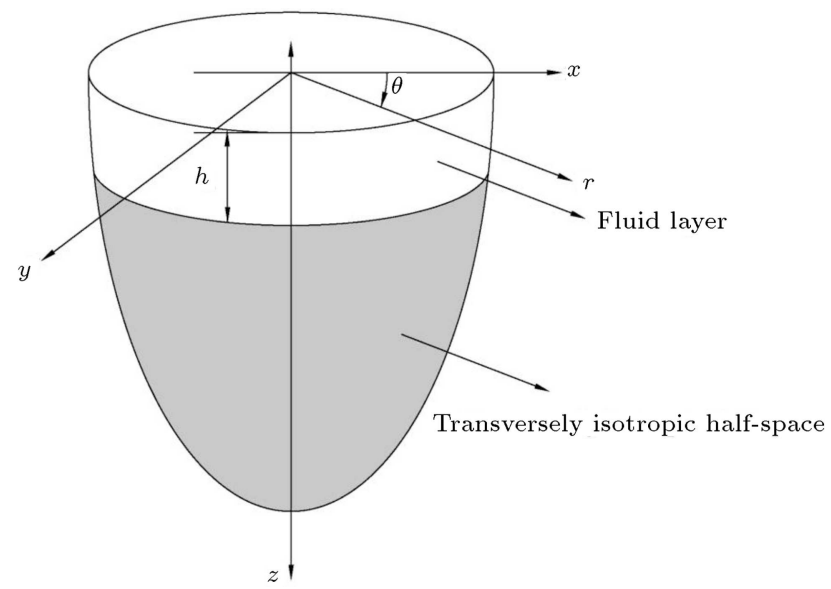

Figure 1. Geometry of the system.

$$
\begin{aligned}
& c_{11}\left(\frac{\partial^{2} u_{r}}{\partial r^{2}}+\frac{1}{r} \frac{\partial u_{r}}{\partial r}-\frac{u_{r}}{r^{2}}\right)+c_{66} \frac{1}{r^{2}} \frac{\partial^{2} u_{r}}{\partial \theta^{2}}-2 c_{11} \frac{1}{r^{2}} \frac{\partial u_{\theta}}{\partial \theta} \\
& +c_{44} \frac{\partial^{2} u_{r}}{\partial z^{2}}+\frac{\left(c_{11}+c_{12}\right)}{2}\left(\frac{1}{r} \frac{\partial^{2} u_{r}}{\partial r \partial \theta}+\frac{1}{r^{2}} \frac{\partial u_{\theta}}{\partial \theta}\right) \\
& +\left(c_{13}+c_{44}\right) \frac{\partial^{2} u_{z}}{\partial r \partial z}+\rho_{s} \omega^{2} u_{r}=0, \\
& c_{66}\left(\frac{\partial^{2} u_{\theta}}{\partial r^{2}}+\frac{1}{r} \frac{\partial u_{\theta}}{\partial r}-\frac{u_{\theta}}{r^{2}}\right)+c_{11} \frac{1}{r^{2}} \frac{\partial^{2} u_{\theta}}{\partial \theta^{2}}+c_{44} \frac{\partial^{2} u_{\theta}}{\partial z^{2}} \\
& +2 c_{11} \frac{1}{r^{2}} \frac{\partial u_{r}}{\partial \theta}+\frac{\left(c_{11}+c_{12}\right)}{2}\left(\frac{1}{r} \frac{\partial^{2} u_{r}}{\partial r \partial \theta}-\frac{1}{r^{2}} \frac{\partial u_{r}}{\partial \theta}\right) \\
& +\left(c_{13}+c_{44}\right) \frac{1}{r} \frac{\partial^{2} u_{z}}{\partial \theta \partial z}+\rho_{s} \omega^{2} u_{\theta}=0, \\
& +\rho_{s} \omega^{2} u_{z}=0, \\
& +\left(\frac{\partial^{2} u_{z}}{\partial r^{2}}+\frac{1}{r} \frac{\partial u_{z}}{\partial r}+\frac{1}{r^{2}} \frac{\partial^{2} u_{z}}{\partial \theta^{2}}\right)+c_{33} \frac{\partial^{2} u_{z}}{\partial z^{2}} \\
& +\left(c_{13}+c_{44}\right) \times\left(\frac{\partial^{2} u_{r}}{\partial r \partial z}+\frac{1}{r} \frac{\partial u_{r}}{\partial z}+\frac{1}{r} \frac{\partial^{2} u_{\theta}}{\partial \theta \partial z}\right)
\end{aligned}
$$

where the time factor $\exp (i \omega t)$ is suppressed. In order to uncouple these equations, a couple of potential functions introduced by Eskandari-Ghadi [21] are applied. These two potential functions, $F$ and $\chi$, are related to displacement components $u_{r}, u_{\theta}$, and $u_{z}$ as:

$$
\begin{aligned}
& u_{r}(r, \theta, z)=-\alpha_{3} \frac{\partial^{2} F(r, \theta, z)}{\partial r \partial z}-\frac{1}{r} \frac{\partial \chi(r, \theta, z)}{\partial \theta}, \\
& u_{\theta}(r, \theta, z)=-\alpha_{3} \frac{1}{r} \frac{\partial^{2} F(r, \theta, z)}{\partial \theta \partial z}+\frac{\partial \chi(r, \theta, z)}{\partial r}, \\
& u_{z}(r, \theta, z)=\left[\left(1+\alpha_{1}\right) \nabla_{r \theta}^{2}+\alpha^{2} \frac{\partial^{2}}{\partial z^{2}}+\frac{\rho_{s} \omega^{2}}{c_{66}}\right] F(r, \theta, z),
\end{aligned}
$$

where:

$$
\nabla_{r \theta}^{2}=\frac{\partial^{2}}{\partial r^{2}}+\frac{1}{r} \frac{\partial}{\partial r}+\frac{1}{r^{2}} \frac{\partial^{2}}{\partial \theta^{2}}
$$

and also:

$$
\alpha_{1}=\frac{c_{12}+c_{66}}{c_{66}}, \quad \alpha_{2}=\frac{c_{44}}{c_{66}}, \quad \alpha_{3}=\frac{c_{13}+c_{44}}{c_{66}} .
$$

Rewriting the equation of motion (Eq. (1)) in terms of the potential functions yields to the two separate partial differential equations as:

$$
\begin{aligned}
& \left(\nabla_{1}^{2} \nabla_{2}^{2}+\delta \omega^{2} \frac{\partial^{2}}{\partial z^{2}}\right) F=0, \\
& \nabla_{0}^{2} \chi=0,
\end{aligned}
$$


defining:

$$
\nabla_{i}^{2}=\nabla_{r \theta}^{2}+\frac{1}{s_{i}^{2}} \frac{\partial^{2}}{\partial z^{2}}+\frac{1}{\mu_{i}} \frac{\rho_{s} \omega^{2}}{c_{66}}, \quad i=0,1,2,
$$

and:

$$
\begin{aligned}
& \mu_{0}=1 \\
& \mu_{1}=\alpha_{2}=\frac{c_{44}}{c_{66}}, \quad \mu_{2}=1+\alpha_{1}=\frac{c_{11}}{c_{66}}, \\
& \frac{\text { delta }}{\rho_{s}}=\left[\frac{-1}{c_{44} s_{2}^{2}}-\frac{1}{c_{11} s_{1}^{2}}+\frac{1}{c_{11}}\left(1+\frac{c_{33}}{c_{44}}\right)\right],
\end{aligned}
$$

where:

$$
s_{0}=\frac{1}{\sqrt{\alpha_{2}}} .
$$

Note that $s_{1}$ and $s_{2}$ are not zero or pure imaginary numbers with respect to the positive definiteness of the strain energy function [22]:

$$
c_{33} c_{44} s^{4}+\left(c_{13}^{2}+2 c_{13} c_{44}-c_{11} c_{33}\right) s^{2}+c_{11} c_{44}=0 .
$$

The two real roots of this equation can be easily obtained since no unknowns of odd order exist in Eq. (9). Hence $s_{1}$ and $s_{2}$ are obtained by Eq. (10) as shown in Box I. With respect to the angular direction, Fourier series expansion is now applied as follows:

$$
[F(r, \theta, z), \chi(r, \theta, z)]=\sum_{-\infty}^{\infty}\left[F_{m}(r, z), \chi_{m}(r, z)\right] e^{i m \theta}
$$

Also, with respect to the radial direction, $m$ th order Hankel transform may be expressed as:

$$
\begin{aligned}
& {\left[\tilde{F}^{m}(\xi, z), \tilde{\chi}^{m}(\xi, z)\right]} \\
& \quad=\int_{0}^{\infty}[F(r, z), \chi(r, z)] r J_{m}(r \xi) d r \\
& \quad=\int_{0}^{\infty}\left[\tilde{F}^{m}(\xi, z), \tilde{\chi}^{m}(\xi, z)\right] \xi J_{m}(r \xi) d \xi .
\end{aligned}
$$

Applying Hankel transform and Fourier series expansion, one may arrive at the two following ordinary differential equations for transformed components of and $F$ and $\chi$ as [9]:

$$
\begin{aligned}
& \left(\tilde{\nabla}_{1 m}^{2} \tilde{\nabla}_{2 m}^{2}+\delta \omega^{2} \frac{d^{2}}{d z^{2}}\right) \tilde{F}_{m}^{m}(z)=0 \\
& \tilde{\nabla}_{0 m}^{2} \tilde{\chi}_{m}^{m}=0
\end{aligned}
$$

in which:

$$
\tilde{\nabla}_{i m}^{2}=\frac{\rho_{s} \omega^{2}}{\mu_{i} c_{66}}-\xi^{2}+\frac{1}{s_{i}^{2}} \frac{d^{2}}{d z^{2}}, \quad i=0,1,2 .
$$

The general solutions for equations in Eq. (13) are:

$$
\begin{aligned}
\tilde{F}_{m}^{m}(\xi, z)= & A_{m}(\xi) e^{\lambda_{1} z}+B_{m}(\xi) e^{-\lambda_{1} z}+C_{m}(\xi) e^{\lambda_{2} z} \\
& +D_{m}(\xi) e^{-\lambda_{2} z} \\
\tilde{\chi}_{m}^{m}(\xi, z)= & E_{m}(\xi) e^{\lambda_{3} z}+F_{m}(\xi) e^{-\lambda_{3} z}
\end{aligned}
$$

introducing:

$$
\begin{aligned}
& \lambda_{1}=\sqrt{a \xi^{2}+b+\frac{1}{2} \sqrt{c \xi^{4}+d \xi^{2}+e}}, \\
& \lambda_{2}=\sqrt{a \xi^{2}+b-\frac{1}{2} \sqrt{c \xi^{4}+d \xi^{2}+e}}, \\
& \lambda_{3}=s_{0} \sqrt{\xi^{2}-\frac{\rho_{s} \omega^{2}}{c_{66}}} .
\end{aligned}
$$

In the above terms:

$$
\begin{aligned}
a= & \frac{1}{2}\left(s_{1}^{2}+s_{2}^{2}\right), \\
b= & -\frac{1}{2} \rho_{s} \omega^{2}\left(\frac{1}{c_{33}}+\frac{1}{c_{44}}\right), \\
c= & \left(s_{2}^{2}-s_{1}^{2}\right)^{2}, \\
d= & -2 \rho_{s} \omega^{2}\left[\left(\frac{1}{c_{33}}+\frac{1}{c_{44}}\right)\left(s_{1}^{2}+s_{2}^{2}\right)\right. \\
& \left.-2 \frac{c_{11}}{c_{33}}\left(\frac{1}{c_{11}}+\frac{1}{c_{44}}\right)\right], \\
e= & \rho_{s}^{2} \omega^{4}\left(\frac{1}{c_{33}}-\frac{1}{c_{44}}\right)^{2} .
\end{aligned}
$$

The unknown functions $A_{m}, B_{m}, C_{m}, D_{m}, E_{m}$, and $F_{m}$ will be determined from the boundary conditions.

$$
s_{1}, s_{2}=\sqrt{\frac{-\left(c_{13}^{2}+c_{13} c_{44}-c_{11} c_{33}\right) \pm \sqrt{\left(c_{13}^{2}+c_{13} c_{44}-c_{11} c_{33}\right)^{2}}-4 c_{33} c_{11} c_{44}^{2}}{2 c_{33} c_{44}}}
$$


$\lambda_{1}, \lambda_{2}$, and $\lambda_{3}$ are multi-valued functions and are made single-valued by specifying the branch cuts emanating from the branch points $\xi_{\lambda_{1}}= \pm \omega \sqrt{\rho_{s} / c_{11}}, \xi_{\lambda_{2}}=$ $\pm \omega \sqrt{ } \rho_{s} / c_{44}$, and $\xi_{\lambda_{3}}= \pm \omega \sqrt{\rho_{s} / c_{66}}$ on the complex $\xi$ plane, such that the real parts of $\lambda_{1}, \lambda_{2}$, and $\lambda_{3}$ remain non-negative for all values of $\xi[10]$. The positive sign of these branch points denotes the wave numbers of the body waves, $\mathrm{P}, \mathrm{SV}$, and $\mathrm{SH}$, respectively. Unlike an isotropic media, transverse isotropy of the medium causes different velocities for $\mathrm{SV}$ and $\mathrm{SH}$ waves in this kind of solids [5]. The Fourier components of the transformed stress and displacement can be expressed as [10]:

$$
\begin{gathered}
\tilde{u}_{z_{m}}^{m}=\left[\alpha_{2} \frac{d^{2}}{d z^{2}}+\frac{\rho_{s} \omega^{2}}{c_{66}}-\xi^{2}\left(1+\alpha_{1}\right)\right] \tilde{F_{m}^{m}}, \\
\tilde{u}_{r_{m}}^{m+1}+i \tilde{u}_{\theta_{m}}^{m+1}=\alpha_{3} \xi \frac{d \tilde{F}_{m}^{m}}{d z}-i \xi \tilde{\chi}_{m}^{m}, \\
\tilde{u}_{r_{m}}^{m-1}-i \tilde{u}_{\theta_{m}}^{m-1}=-\alpha_{3} \xi \frac{d \tilde{F}_{m}^{m}}{d z}-i \xi \tilde{\chi}_{m}^{m}, \\
\tilde{\sigma}_{z r_{m}}^{m+1}+i \tilde{\sigma}_{z \theta_{m}}^{m+1}=c_{44} \xi\left[\left(\alpha_{3}-\alpha_{2}\right) \frac{d^{2}}{d z^{2}}+\xi^{2}\left(1+\alpha_{1}\right)\right. \\
\left.-\frac{\rho_{s} \omega^{2}}{c_{66}}\right] \tilde{F}_{m}^{m}-c_{44} \xi i \frac{d \tilde{\chi}_{m}^{m}}{d z}, \\
\left.\quad-\frac{\rho_{s} \omega^{2}}{c_{66}}\right] \tilde{F}_{m}^{m}-c_{44} \xi i \frac{d \tilde{\chi}_{m}^{m}}{d z} .
\end{gathered}
$$

For the compressible liquid, the governing equation of time-harmonic motion in cylindrical coordinate system is [1]:

$$
\frac{K}{\rho_{l}} \nabla^{2} \varphi(r, \theta, z)=-\omega^{2} \varphi
$$

where $\varphi, K$, and $\rho_{l}$ are the displacement potential, bulk modulus, and density of the liquid, respectively. The displacement components and pressure can now be expressed in terms of $\varphi$ as:

$$
\begin{aligned}
& u_{r}=\frac{\partial \varphi}{\partial r}, \quad u_{z}=\frac{\partial \varphi}{\partial z}, \quad u_{\theta}=\frac{1}{r} \frac{\partial \varphi}{\partial \theta} \\
& P=-K \nabla^{2} \varphi=\rho_{l} \omega^{2} \varphi
\end{aligned}
$$

Similar to the solid substratum, the procedure of Fourier series expansion is followed with respect to the angular direction, and applying $m$ th-order Hankel transform is followed with respect to the radial direction:

$$
\begin{aligned}
& \varphi(r, \theta, z)=\sum_{-\infty}^{\infty} \varphi_{m}(r, z) e^{i m \theta}, \\
& \tilde{\varphi}^{m}(\xi, z)=\int_{0}^{\infty} \varphi(r, z) r J_{m}(r \xi) d r,
\end{aligned}
$$

where the inverse transform can be used to return to the real domain as:

$$
\varphi(r, z)=\int_{0}^{\infty} \tilde{\varphi}^{m}(\xi, z) \xi J_{m}(r \xi) d \xi .
$$

The same is followed for stress and displacement components. The respective ordinary differential equation of motion in terms of $\tilde{\varphi}_{m}^{m}$ can be obtained as:

$$
\left(\frac{\rho_{l} \omega^{2}}{K}-\xi^{2}+\frac{d^{2}}{d z^{2}}\right) \tilde{\varphi}_{m}^{m}=0 .
$$

A general solution to this equation is:

$$
\tilde{\varphi}_{m}^{m}=S_{m}(\xi) e^{-\lambda_{4} z}+R_{m}(\xi) e^{\lambda_{4} z},
$$

where:

$$
\lambda_{4}=\sqrt{\xi^{2}-\frac{\omega^{2}}{c_{l}^{2}}} .
$$

Here, $c_{l}=\sqrt{K / \rho_{l}}$ is the velocity of the compressional wave in the liquid, and $S_{m}$ and $R_{m}$ will be calculated from the boundary conditions, where $R_{m}$ is associated with the amplitude of the transmitting wave from the bottom boundary, and $S_{m}$ is associated with the reflecting wave amplitude from the free surface of the liquid.

Fourier factors of stress and displacement component in the transformed mode are written as:

$$
\begin{aligned}
& \tilde{\sigma}_{z z}=-\tilde{P}=-\rho_{l} \omega^{2} \tilde{\varphi}_{m}^{m} \\
& \tilde{u}_{r_{m}}^{m}=\xi\left[\frac{m+1}{2 m} \tilde{\varphi}_{m}^{m-1}-\frac{m-1}{2 m} \tilde{\varphi}_{m}^{m+1}\right], \\
& \tilde{u}_{z_{m}}^{m}=\frac{\partial \tilde{\varphi}_{m}^{m}}{\partial z} \\
& \tilde{u}_{\theta_{m}}^{m}=\frac{i \xi}{2}\left(\tilde{\varphi}_{m}^{m-1}+\tilde{\varphi}_{m}^{m+1}\right) .
\end{aligned}
$$

\section{Boundary conditions and frequency equation}

The boundary conditions for the problem under consideration are given below. The expressions provided in Eqs. (18) along with Eqs. (27) are utilized. The coefficients $A_{m}, C_{m}$, and $E_{m}$ are omitted according to the radiation condition.

The free surface condition requires the vanishing of the liquid pressure, $P$ : 


$$
\sigma_{z z}(z=0)=0 .
$$

This imposes:

$$
S_{m}(\xi)=-R_{m}(\xi)
$$

At the interface of the solid and liquid, i.e. at $z=h$, the boundary conditions imply continuity of the normal component of the displacements of the two media:

$$
u_{z}\left(z=h^{-}\right)=u_{z}\left(z=h^{+}\right),
$$

which means:

$$
\begin{gathered}
\left(e^{-\lambda_{1} h}\right)\left[\alpha_{2} \lambda_{1}^{2}+\left(\frac{\rho_{s} \omega^{2}}{C_{66}}-\xi^{2}\left(1+\alpha_{1}\right)\right)\right] B_{m}(\xi) \\
+\left(e^{-\lambda_{2} h}\right)\left[\alpha_{2} \lambda_{2}^{2}+\left(\frac{\rho_{s} \omega^{2}}{c_{66}}-\xi^{2}\left(1+\alpha_{1}\right)\right)\right] \\
D_{m}(\xi)+\lambda_{4}\left(S_{m}(\xi) e^{-\lambda_{4} h}-R_{m}(\xi) e^{\lambda_{4} h}\right)=0
\end{gathered}
$$

Meanwhile, since the liquid does not support shear stresses, the two tangential stress components, $\sigma_{z \theta}$ and $\sigma_{r z}$, vanish at the interface:

$$
\begin{gathered}
\sigma_{z \theta}(z=h)=0, \\
\sigma_{r z}(z=h)=0 .
\end{gathered}
$$

These two conditions yield to the following equations, respectively:

$$
\begin{gathered}
{\left[2 C_{44} \xi i \lambda_{3} e^{-\lambda_{3} h}\right] F_{m}(\xi)=0} \\
\left\{\left[2 C_{44}\left(\alpha_{3}-\alpha_{2}\right) \lambda_{1}^{2}+\xi^{2}\left(1+\alpha_{1}\right)-\frac{\rho_{s} \omega^{2}}{c_{66}}\right] e^{-\lambda_{1} h}\right\} B_{m}(\xi) \\
+\left\{\left[2 c_{44}\left(\alpha_{3}-\alpha_{2}\right) \lambda_{2}^{2}+\xi^{2}\left(1+\alpha_{1}\right)\right.\right. \\
\left.\left.-\frac{\rho_{s} \omega^{2}}{c_{66}}\right] e^{-\lambda_{2} h}\right\} D_{m}(\xi)=0
\end{gathered}
$$

The normal stress continuity is preserved at the solidliquid interface:

$$
\begin{aligned}
\sigma_{z z}\left(z=h^{-}\right)= & \sigma_{z z}\left(z=h^{+}\right) \\
{\left[\alpha_{3} c_{13} \xi^{2}\right.} & \left.+c_{33}\left(\frac{\rho_{s} \omega^{2}}{c_{66}}-\xi^{2}\left(1+\alpha_{1}\right)\right)+c_{33} \alpha_{2} \lambda_{1}^{2}\right] \\
& \left(-\lambda_{1} e^{-\lambda_{1} h}\right) B_{m}(\xi)+\left[\alpha_{3} c_{13} \xi^{2}\right. \\
& \left.+c_{33}\left(\frac{\rho_{s} \omega^{2}}{C_{66}}-\xi^{2}\left(1+\alpha_{1}\right)\right)+c_{33} \alpha_{2} \lambda_{2}^{2}\right] \\
& \left(-\lambda_{2} e^{-\lambda_{2} h}\right) D_{m}(\xi)-\left[( \rho _ { s } \omega ^ { 2 } ) \left(S_{m} e^{-\lambda_{4} h}\right.\right. \\
& \left.\left.+R_{m} e^{\lambda_{4} h}\right)\right] S_{m}(\xi)=0 .
\end{aligned}
$$

Two separate sets of equations for the five remaining unknown functions, $B_{m}, D_{m}, F_{m}, S_{m}$, and $R_{m}$, are now provided. These equations constitute the matrix given by Eq. (38) as shown in Box II, where:

$$
\begin{aligned}
\eta_{i} & =\left(\alpha_{3}-\alpha_{2}\right) \lambda_{1}^{2}+\xi^{2}\left(1+\alpha_{1}\right)-\frac{\rho_{s} \omega^{2}}{c_{66}}, \\
\vartheta_{i} & =\alpha_{3} \lambda_{i}^{2}-\eta_{i}, \\
\nu_{i} & =\left(\eta_{i}-\alpha_{3} \frac{c_{13}}{c_{33}} \xi^{2}-\alpha_{3} \lambda_{i}^{2}\right) \lambda_{i}, \quad i=1,2 .
\end{aligned}
$$

The nontrivial solution is obtained when the determinant of the matrix vanishes, that is:

$$
\operatorname{det}[A]=0 \text {. }
$$

The expression for $\chi_{m}$ is separated from the other unknowns and reduces to $\lambda_{3}=0$. According to the assumed non-viscos nature of the liquid, no Love wave is expected to appear at the interface which is mathematically observed here, since no roots are obtained in the expression given for $\chi_{m}$, except one determining the $\mathrm{SH}$ wave-number.

Eq. (40) provides the frequency equation with respect to the two-layer half-space; it relates $\xi$, the wavenumber, to $\omega$ which is the angular frequency; it presents the dispersive properties of the system. For any value of $\omega$, there is at least one root, $\xi_{R}>\max \left(\xi_{\lambda_{1}}, \xi_{\lambda_{2}}\right)$, associated with the Rayleigh wave-number which satisfies the frequency equation. Dependent on the liquid layer

$$
A=\left[\begin{array}{cccc}
e^{-\lambda_{1} h} \vartheta_{1} & e^{-\lambda_{2} h} \vartheta_{2} & \lambda_{4} e^{-\lambda_{4} h} & -\lambda_{4} e^{-\lambda_{4} h} \\
e^{-\lambda_{1} h} c_{33} \nu_{1} & e^{-\lambda_{2} h} c_{33} \nu_{2} & -\rho_{s} \omega^{2} e^{-\lambda_{4} h} & -\rho_{s} \omega^{2} e^{-\lambda_{4} h} \\
2 \xi c_{44} \eta_{1} e^{-\lambda_{1} h} & 2 \xi c_{44} \eta_{2} e^{-\lambda_{2} h} & 0 & 0 \\
0 & 0 & 1 & -1
\end{array}\right]
$$


depth, frequency of the excitations, and properties of the liquid and bed material, there might be other roots which are associated with the higher modes. Notwithstanding, only a single root may exceed the value $\omega / c_{l}$ which will be associated with the Stoneleyor Scholte-wave-number. If the compressional wave velocity of the liquid is higher than the SV wave velocity, the only possible root will be related to the Stoneley wave and no higher modes will appear.

\section{Special cases}

To draw a comparison between the results obtained here with some existing ones, several special cases of the problem are now considered:

- Case 1. If $\xi h$ tends to infinity or equivalently the liquid layer depth takes very large values compared to the wavelength, the velocity of the surface waves tends to the Stoneley - or Scholte-wave velocity, propagating in a bi-material full space consisting of liquid and transversely isotropic solid half-spaces. This results in the wave-number, $\xi_{s t}$, of Stoneley wave showing exact agreement with the frequency equation presented by Abubakar and Hudson [15]:

$$
\rho_{l} \omega^{2}\left(\eta_{2} \vartheta_{1}-\eta_{1} \vartheta_{2}\right)+c_{33} \lambda_{4}\left(\eta_{2} \nu_{1}-\eta_{1} \nu_{2}\right)=0 \text {. }
$$

- Case 2. If $\xi h$ tends to zero, meaning that the wave-length becomes very large in comparison to the depth of the liquid layer, the frequency equation reduces to that of a Rayleigh wave for a transversely isotropic solid half-space developed by Khojasteh et al. [10], i.e. $\eta_{1} \nu_{2}-\eta_{2} \nu_{1}=0$.

- Case 3. If the solid material is considered to be isotropic, the wave-numbers associated with the body waves become $\xi_{P}=\omega \sqrt{\rho_{s} /(2 \mu+\lambda)}$ and $\xi_{\mathrm{SH}}=$ $\xi_{\mathrm{SV}}=\omega \sqrt{\rho_{s} / \mu}$, and the frequency equation reduces to the equation developed by Ewing [3] for an isotropic bed material underlying a liquid layer.

- Case 4. If the solid material is reduced to an isotropic medium and $\xi h$ tends to zero, the Rayleigh wave velocity for isotropic half-space is obtained. As a more specific case, where $\lambda=\mu$, the well-known function for Rayleigh wave velocity is deduced [10] as: $c_{R}=\frac{2}{\sqrt{3+\sqrt{3}}} \sqrt{\frac{\mu}{\rho_{s}}}$. Also, if the solid is simplified to an isotropic case and $\xi h$ becomes very large, the interface Stoneley wave is obtained for a full-space bi-materials system consisting of liquid and isotropic solid half-spaces. These special cases are numerically verified in the following section.

\section{Numerical results and discussion}

The derived frequency equation in the previous section implicitly expresses the wave-number, $\xi$, in terms of $\omega$,
Table 1. Material properties.

\begin{tabular}{cccccccc}
\hline Material & $\boldsymbol{E}_{\boldsymbol{v}} / \boldsymbol{E}_{\boldsymbol{h}}$ & $\boldsymbol{\nu}_{\boldsymbol{h}}$ & $\overline{\boldsymbol{c}}_{\mathbf{1 1}}$ & $\overline{\boldsymbol{c}}_{\mathbf{1 2}}$ & $\overline{\boldsymbol{c}}_{\mathbf{1 3}}$ & $\overline{\boldsymbol{c}}_{\boldsymbol{3 3}}$ & $\boldsymbol{\varepsilon}(\boldsymbol{\%})$ \\
\hline $1^{\mathrm{a}}$ & 1 & 0.25 & 3.00 & 1.00 & 1.00 & 3.00 & 0 \\
$2^{\mathrm{b}}$ & 2 & 0.33 & 3.00 & 1.12 & 1.03 & 5.51 & 22.78 \\
$3^{\mathrm{b}}$ & 3 & 0.17 & 2.65 & 0.52 & 0.79 & 7.91 & 33.25 \\
$4^{\mathrm{b}}$ & 4 & 0.25 & 2.72 & 0.72 & 0.86 & 12.93 & 39.48 \\
\hline
\end{tabular}

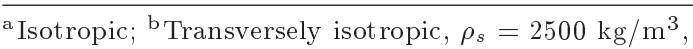

$\bar{c}_{i j}=c_{i j} / c_{44}$, and $c_{44}=10 \mathrm{GPa}$.

which provides the surface waves velocity. By means of a computer program written in Mathematica, the roots of the equation are calculated and the dispersion curves are obtained consequently. The non-dimensional phase and group velocities of the surface wave are plotted versus another dimensionless variable, $\gamma=\xi_{n} c_{n} h / 2 \pi c_{l}$, in semi-logarithmic figures. Four solid materials, whose elastic properties are presented in Table 1, are considered as the underlying medium. The elastic constants are normalized with respect to $c_{44}$. In this table, the materials are sorted with respect to the anisotropy parameter, $\varepsilon$, which is defined as follows [23]:

$$
\varepsilon=\frac{c_{11}-c_{33}}{2 c_{33}} \times 100
$$

Material 1, shown in Table 1, is an isotropic material; hence, its anisotropy parameter is equal to zero. In all configurations, the liquid is taken to be water with the properties: $\rho_{l}=1000 \mathrm{~kg} / \mathrm{m}^{3}$ and $K=2.2 \mathrm{GPa}$.

The positive definiteness of the strain energy is considered when defining the material. The elasticity constants should comply with the requirements below [21]:

$$
c_{11}>\left|c_{12}\right|, \quad\left(c_{11}+c_{12}\right) c_{33}>2 c_{13}^{2}, \quad c_{44}>0 .
$$

The phase velocity associated with the first mode, and the next three higher modes for systems consisting of half-space of kind Materials 2 and 4 (Table 1) underlying a water layer are provided in Figures 2

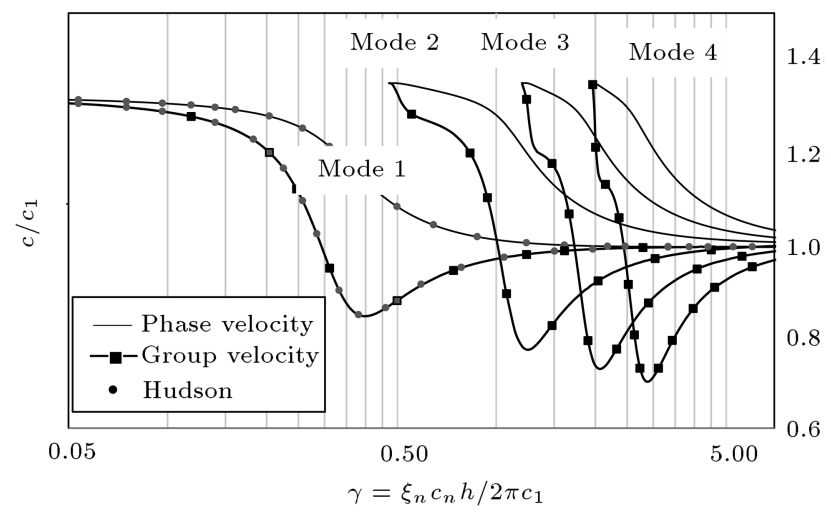

Figure 2. Phase and group velocities for the first four modes of Material 4 underlying a water. 


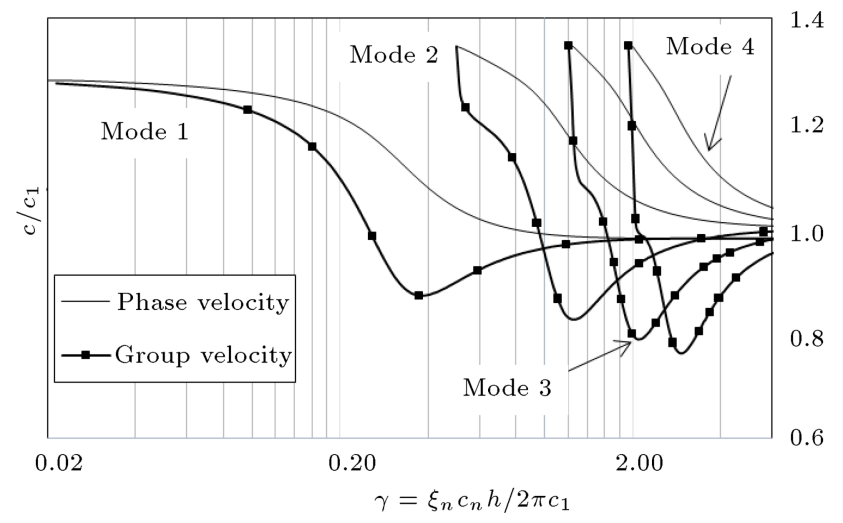

Figure 3. Phase and group velocities for the first four modes of Material 2 underlying a water layer.

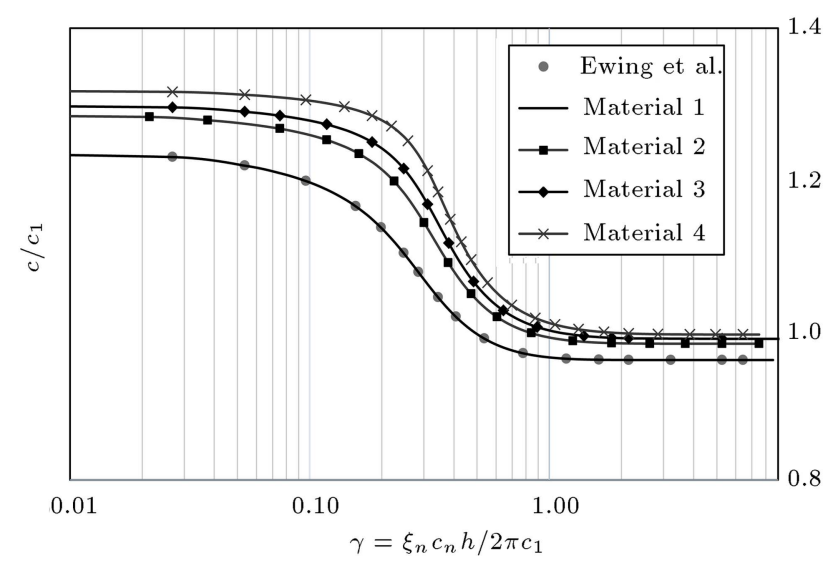

Figure 4. Phase velocity of the first modes for four bed materials as the bed.

and 3. The results show exact agreement with those presented by Abubakar and Hudson [15]. It can be observed that the higher modes take a maximum value of cutoff phase velocity equal to the velocity of SV wave propagation in the solid substratum, i.e. $c_{\mathrm{SV}}=$ $\sqrt{c_{44} / \rho_{s}}$. Nevertheless, for the first mode, it takes the Rayleigh wave velocity for the respective vacuum-solid half-space system.

The dimensionless phase velocities of the first modes of three transversely isotropic materials along with one isotropic material underlying a finite water layer are shown in Figure 4. It can be observed that the curve for the isotropic material is in exact agreement with the results presented by Ewing et al. [3]. An isotropic solid can be degenerated from a transversely isotropic one appropriately setting the elasticity constants as $c_{11}=c_{33}=\lambda+2 \mu, c_{12}=$ $c_{13}=\lambda$, and $c_{44}=c_{66}=\mu$. In an isotropic material, $\lambda_{1}=\sqrt{\xi^{2}-\xi_{P}^{2}}$, and $\lambda_{2}=\lambda_{3}=\sqrt{\xi^{2}-\xi_{S}^{2}}$.

As it is apparent in Figures 2, 3, and 5, all values of the phase velocities for higher modes fall above the value $c_{l}$, whereas, for the normal mode, there are values of the velocity less than $c_{1}$ which tend to the asymptotic value of Stoneley or Scholte wave velocity. Stoneley

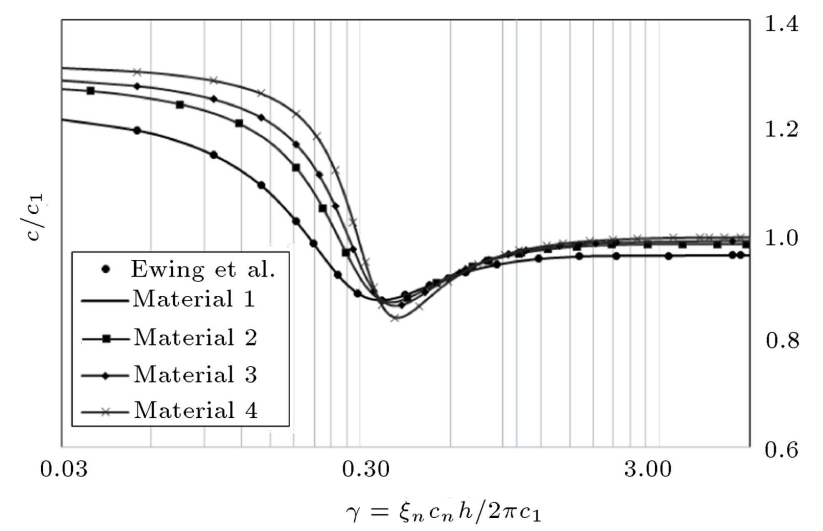

Figure 5. Group velocities of the first modes for four bed materials as the bed underlying a layer of water.

waves can always arise in the systems of contacting liquid and solid media. Nevertheless, it should be noted that in contacting solid layers, the possibility of this type of surface waves depends on the properties of the layers [3]. The Stoneley branch of the curve arises in a higher value of $\xi h$ as the elastic constants of the material increase. Normal mode group velocities for the defined materials as the solid half-space are plotted in Figure 5 for comparison. Unlike the phase velocity, the group velocity takes a minimum value (Airy phase) which occurs at a higher $\xi h$ value in the higher modes. All the figures indicate that the velocities of SV wave in the solid domain and the $P$ wave in the liquid mainly control the range of the values that the dispersion curves covers.

\section{Summary and conclusion}

In this paper, dispersion of generalized Rayleigh waves is studied in a model of a homogeneous compressible finite liquid layer lying over a transversely isotropic solid half-space. Hankel transform and Fourier series expansion along with the method of potential functions are used to deal with the equations of motion. The dispersive nature of the surface waves is described through the derived frequency equation. Various special cases are studied to confirm the formulation and numerical results with the existing solutions. In this regard, both the mathematical formulation and numerical results are derived for several simpler half- and full spaces degenerated from the most general case. The results are compared and confirmed with the existing ones. The figures clearly show that the material anisotropy can play an important role in the dispersive characteristics of surface waves. The considerable influence of the liquid layer depth relative to the wave-length, as well as the bed material properties, on the characteristics of the phenomenon as well as the elastic properties of the bed can be observed from the figures presented for different bed materials. The velocity dispersion curves 
reveal the fact that the difference between the velocities of SV and SH waves in a VTI solid is important for the lower limit of the velocities of higher modes. As in this case, the lower limit is the $\mathrm{SV}$ wave velocity which is different from the $\mathrm{SH}$ wave velocity. This indicates the importance of the anisotropy, as the SV and $\mathrm{SH}$ wave velocities may significantly differ in the highly anisotropic materials, and hence in the velocity dispersion curves. Consequently, neglecting the effect of the anisotropy may clearly results in considerable inaccuracies.

The obtained results can be efficiently extended to calculate the elastodynamic response of described system subjected to dynamic excitations which is being processed by the authors and can be effectively applied to solve bed-fluid-structure problems.

\section{Nomenclature}

c Dispersive wave velocity;

$c_{i j} \quad$ Elasticity constants;

$c_{l} \quad$ Velocity of compressional wave in the liquid;

$c_{n} \quad n$th mode interface wave velocity;

$c_{R} \quad$ Rayleigh wave velocity;

$c_{\mathrm{SV}} \quad \mathrm{SV}$ wave velocity;

$E_{h} \quad$ Young's moduli in the direction normal to the plane of transverse isotropy;

$E_{v} \quad$ Young's moduli in the plane of transverse isotropy;

$F \quad$ Potential function;

$h \quad$ Liquid layer depth;

$J_{m} \quad$ Bessel function of the first kind and $m$ th order;

$K \quad$ Liquid bulk modulus;

$k_{p} \quad$ Dilatational wave-number;

$k_{s} \quad$ Distortional wave-number;

$m \quad$ Hankel integral transform order;

$P \quad$ Liquid dynamic pressure;

$r \quad$ Radial coordinate;

$s_{1}, s_{2} \quad$ Roots of strain energy function;

$t \quad$ Time variable;

$u_{r} \quad$ Displacement component in $r$-direction;

$u_{z} \quad$ Displacement component in $z$ direction;

$u_{\theta} \quad$ Displacement component in $\theta$ direction;

$\varepsilon \quad$ Anisotropy parameter;

$\theta \quad$ Angular coordinate;

$\lambda \quad$ Lame's constant;

$\mu \quad$ Lame's constant; $\nu_{h} \quad$ Poisson ratio characterizing the effects of horizontal strain on complementary horizontal strain;

$\xi \quad$ Hankel's parameter and wave-number;

$\xi_{R} \quad$ Rayleigh wave-number;

$\xi_{s t} \quad$ Stoneley wave-number;

$\xi_{n} \quad n$th Mode wave-number;

$\rho_{l} \quad$ Liquid density;

$\rho_{s} \quad$ Solid density;

$\sigma_{i j} \quad$ Stress components;

$\chi \quad$ Potential function;

$\omega \quad$ Frequency.

\section{References}

1. Stoneley, R. "The effect of ocean on Rayleigh waves", Mon. Not. R. Astro. Soc. Geophys. Suppl., 1, pp. 349356 (1926).

2. Haskell, N.A. "The dispersion of surface waves in multilayered media", Bull. Sesmol. Soc. Am., 43, pp. 17-34 (1953).

3. Ewing, W.M., Jardetzky, W.S. and Press, F., Elastic Waves in Layered Media, McGraw-Hill Book Company, INC., New York, Toronto, London (1957).

4. Crampin, S. "Review of wave motion in anisotropic and cracked elastic media", Wave Motion, 3, pp. 343391 (1981).

5. Stoneley, R. "The seismological implications of aeolotropy in continental structures", Roy. Astron. Soc. Mon. Notices, Geophysical Supplement, 5, pp. 343-353 (1949).

6. Synge, J.L. "Elastic waves in anisotropic media", $J$. Math. Phys., 35, pp. 323-334 (1957).

7. Buchwald, V.T. "Rayleigh waves in transversely isotropic media", Q. J. Mech. Appl. Math., 14(4), pp. 293-317 (1961).

8. Payton, R.G., Elastic Wave Propagation in Transversely Isotropic Media, Martinus, Nijhoff, the Netherlands (1983).

9. Rahimian, M., Eskandari-Ghadi, M., Pak, R.Y.S. and Khojasteh, A. "Elastodynamic potential method for transversely isotropic solid", J. Eng. Mech, ASCE, 133(10), pp. 1134-1145 (2007).

10. Khojasteh, A., Rahimian, M., Eskandari, M. and Pak, R.Y.S. "Asymmetric wave propagation in a transversely isotropic half-space in displacement potentials", Int. J. Eng. Sci., 46, pp. 690-710 (2008).

11. Khojasteh, A., Rahimian, M., Pak, R.Y.S. and Eskandari, M. "Asymmetric dynamic Green's functions in a two-layered transversely isotropic half-space", J. Eng. Mech. ASCE, 134(9), pp. 777-787 (2008).

12. Khojasteh, A., Rahimian, M. and Pak, R.Y.S. "Threedimensional dynamic Green's functions in transversely isotropic bi-materials", Int. J. Solids. Struct., 45, pp. 4952-4972 (2008b). 
13. Khojasteh, A., Rahimian, M. and Eskandari, M. "Three-dimensional dynamic Green's functions in transversely isotropic tri-materials", J. Math. Model., 37, pp. 3164-3180 (2013).

14. Khojasteh, A., Rahimian, M., Eskandari, M. and Pak, R.Y.S. "Three-dimensional dynamic Green's functions for a multilayered transversely isotropic half-space", Int. J. Solids. Struct., 48, pp. 1349-1361 (2011).

15. Abubakar, I. and Hudson, J.A. "Dispersive properties of liquid overlying an aelotropic half-space", Geophys. J. Roy. Astr. Soc., 5, pp. 217-229 (1961).

16. Sharma, M.D., Kumar, R. and Gogna, M.L. "Surface wave propagation in a liquid saturated porous solid layer overlying a homogeneous transversely isotropic half-space and lying under a uniform layer of liquid", Int. J. Solids. Struct., 127, pp. 1255-1267 (1991).

17. Sharma, M.D., Kumar, R. and Gogna, M.L. "Surface wave propagation in a transversely isotropic elastic layer overlying a liquid saturated porous solid halfspace and lying under the uniform layer of liquid", Pure. Appl. Geophys., 133(3), pp. 523-539 (1990).

18. Sharma, M.D. "Dispersion in oceanic crust during earthquake preparation", Int. J. Solids. Struct., 36, pp. 3469-3482 (1999).

19. Kumar, R. and Miglani, A. "Surface wave propagation in an oceanic crust model", Acta Geophys. Polonica, 52(4), pp. 443-456 (2004).

20. Kumar, R. and Kumar, R. "Analysis of wave motion in transversely isotropic elastic material with voids under an inviscid liquid layer", Can. J. Phys., 87(4), pp. 377388 (2009).

21. Eskandari-Ghadi, M. "A complete solutions of the wave equations for transversely isotropic media", $J$. Elast., 81, pp. 1-19 (2005).

22. Lekhnitskii, S.G., Theory of Anisotropic Elastic Bodies, Holden-Day, San Francisco (1963).

23. Ben-Menahem, A. and Sena, A.G. "Seismic source theory in stratified Anisotropic Media", J. Geophyis. Res., 95, pp. 15, 395-15,427 (1990).

\section{Biographies}

Amirhossein Bagheri received his BSc degree in Civil Engineering from Sharif University of Technology in 2008, MSc degree in Hydraulic Structures Engineering from University of Tehran in 2011. He is currently a $\mathrm{PhD}$ candidate of Hydraulic Structures Engineering in University of Tehran, working in fields such as wave propagation and soil-water-structure interactions.
Three contributions have been published by him in international journals and conferences. He was also ranked 383rd among more than 800,000 applicants participated in the nationwide undergraduate program entrance exam. He has published five papers in scientific journals and also several conference papers.

Ali Khojasteh, currently an Assistant Professor in the University of Tehran, received his BSc in Civil Engineering from the University of Shiraz, Iran in 2003, and MSc and PhD degrees from the University of Tehran, Iran, in 2005 and 2009, respectively, both ranked 1 st among graduates.

His research interests are computational mechanics, wave propagation in solids/fluids, mechanics of composites, BEM, soil-structure Interaction. He was ranked 262nd among more than 800,000, 7th among more than 15000, and 9th among more than 15000 participating in the nationwide undergraduate, MSc program entrance exams and the Civil Engineering Olympiad, respectively. More than 15 contributions have been published in the international journals by him.

Mohammad Rahimian, currently the Full Professor of Civil Engineering Department at the University of Tehran, received his BSc degree in Civil Engineering from the University of Tehran. He received his PhD degree in Ecole National Des Ponts et Chaussees, France, in 1981. His research fields are earthquake engineering, wave propagation in anisotropic materials, soil-structure-fluid interaction, etc. $\mathrm{He}$ is the former chair and assistant to chair of the University of Tehran and the former chair of the Faculty of Engineering of the University of Tehran. Near 50 papers published in ISI indexed journals and 6 written or translated books are of his scientific works.

Reza Attarnejad received his $\mathrm{PhD}$ degree in 1990 from UPC in Spain focused on computational mechanics on continuous media. He is currently the (full) Professor of Structural Engineering Division of Civil Engineering Department at University of Tehran and also was the former chairman of the division. $\mathrm{He}$ has extensive publications on his specialties which are fluid-structure interaction, structural dynamics, FEM, applied mathematics, and semi-analytical methods. He has punlished near 70 papers in the ISI indexed international journals. 